\title{
Dynamic Load Distribution in Cooperative Manipulation Tasks
}

\author{
Andrea Zambelli Bais ${ }^{1}$,Sebastian Erhart $^{2}$,Luca Zaccarian ${ }^{3}$,Sandra Hirche ${ }^{2}$
}

\begin{abstract}
In cooperative manipulation tasks, load allocation is a crucial step in order to solve the intrinsic redundancy of the system. The desired wrench needs to be suitably distributed between the end-effectors to implement the desired motion of the manipulated object. In this framework, both the grasp kinematics and the individual capacities of each manipulator provide relevant constraints. On one hand, the end effector wrenches act on the object via the grasp geometry. On the other hand, the individual admissible payload further depends on the current configuration of the robots. In this paper we focus on a heterogeneous cooperative manipulation setting and we design a proper allocation strategy to distribute the desired object wrench, considering both constant and time-varying constraints for the load distribution. The relevance of our findings is illustrated by means of an experimental study involving two anthropomorphic robots manipulating a common object.
\end{abstract}

\section{INTRODUCTION}

Cooperative manipulation of a common object enhances significantly the manipulation task capacity of the manipulator team in terms of payload and dexterity. Potential application areas of cooperating manipulators are manufacturing, construction, agriculture, and search and rescue. The benefits obtained by employing a team of robotic manipulators come at the cost of an increased complexity for the robot coordination. There exists an infinite number of combinations for the individually allocated manipulator load, given a desired force/torque applied to the manipulated object. The load distribution strategy resolves the intrinsic redundancy occurring in cooperative manipulation tasks and allocates suitable force/torque setpoints for the manipulators.

The load distribution problem in robotic manipulation tasks can be considered as a control allocation problem for an overactuated mechanical system [1]. In cooperative manipulation, the load distribution problem is equivalent to the computation of suitable setpoints for the force control schemes of the individual manipulator such as impedance control schemes [2]. The authors of [3] formulate the load distribution as a quadratic optimization problem with equality constraint. The available degrees of freedom for the load allocation are commonly interpreted in terms of motion-inducing and

\footnotetext{
${ }^{1}$ A. Zambelli is with Dipartimento di Ingegneria Industriale, University of Trento, via Sommarive 9, 38123 Trento, Italy zambellieitr.ei.tum.de

${ }^{2} \mathrm{~S}$. Erhart and S. Hirche are with the Chair of Information-oriented control, Technische Universität München, Arcisstraße 21, D-80290 München, Germany $\{$ erhart, hirche\}@tum. de

${ }^{3}$ L. Zaccarian is with CNRS, LAAS, 7 avenue du Colonel Roche, F-31400 Toulouse, France and Université de Toulouse, 7 avenue du Colonel Roche, 31077 Toulouse cedex 4, France, and with Dipartimento di Ingegneria Industriale, University of Trento, Italy. luca.zaccarianeunitn.it
}

internal wrench components, which lead the authors of [4][6] to conclude on the existence of a specific non-squeezing load distribution avoiding internal stress applied to the object. The work in [7] proposes a different result for the nonsqueezing load distribution, rising the question about which load distribution is the one without internal object loading. Recently, a novel interpretation of internal stress as violation of the kinematic constraints gives a physically motivated explanation that there exists no unique non-squeezing load distribution [8]. This new paradigm for the characterization of internal stress offers additional degrees of freedom for choosing a suitable load distribution, allowing one to incorporate heterogeneous payload capacities. Moreover, the admissible payload of an individual robotic arm depends in general on the current manipulator posture, constraining the magnitude and potentially also the rate of the admissible endeffector payload. An input allocation scheme for redundant control systems accounting for magnitude and rate limits of the actuators is presented in [9].

In this paper we present a novel load distribution scheme for cooperative multi-robot manipulation tasks that enables the allocation of heterogeneous end-effector payloads for a manipulator ensemble. We present an intuitive tuning strategy for balancing the payload between individual manipulators in terms of weighting coefficients. For a particular case, we give an explicit expression of the solution of the corresponding optimization problem. In view of a dynamic load allocation strategy, we present a consistent manipulator payload model assuming constant joint torques. We treat the emerging magnitude-saturation of the manipulators as a system with slowly time-varying parameters and design a proper dynamic allocator. The presented findings are evaluated by means of an experimental study on two cooperating manipulators.

The remainder of this paper is organized as follows. In Section II the general problem setting is presented. Section III and IV deal with the static and dynamic load distribution problem, respectively. Experimental results are presented in Section V and a conclusion is given in Section VI.

\section{PROBLEM SETTING}

Consider $N$ manipulators grasping a rigid object. In the sequel we assume a rigid grasp, i.e. no end-effector slippage occurs. Then the relationship between the force/torque $h_{o}^{d}$ applied to the object and the end-effector wrenches $h^{d}$ corresponds to:

$$
h_{o}^{d}=G h^{d},
$$

wherein $h_{o}^{d}=\left(f_{o}^{d^{T}}, t_{o}^{d^{T}}\right)^{T} \in \mathbb{R}^{6}$ is the desired wrench applied to the origin of the object frame $O$, with the force 
and torque components $f_{i}$ and $t_{i} \in \mathbb{R}^{3}$ for $i \in\{1, \ldots, N\}$ respectively. We assume that the desired object wrench is given a priori, e.g. by specifying the object trajectory and computing $h_{o}^{d}$ by the object's inverse dynamics as e.g. done in standard impedance control design approaches [10]. $h^{d}=\left(h_{1}^{d^{T}}, \ldots, h_{N}^{d}{ }^{T}\right)^{T} \in \mathbb{R}^{6 N}$ is the stacked vector of the end-effector wrenches and $G \in \mathbb{R}^{6 \times 6 N}$ is the grasp matrix [11] defined by means of the end-effector grasp points $r_{i} \in \mathbb{R}^{3}$, as follows:

$$
G=\left[\begin{array}{ccccc}
I_{3} & 0_{3} & \cdots & I_{3} & 0_{3} \\
S\left(r_{1}\right) & I_{3} & \cdots & S\left(r_{N}\right) & I_{3}
\end{array}\right],
$$

where $S(\cdot)$ is a skew-symmetric matrix performing the crossproduct, i.e. $S(u) w=u \times w$ for $u, w \in \mathbb{R}^{3}$. If not stated otherwise, all quantities are expressed in the objectfixed coordinate frame $O$. This coordinate frame and the manipulation task kinematics are illustrated in Fig. 1.

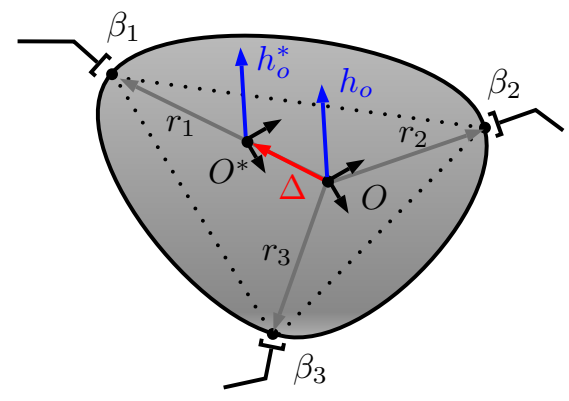

Fig. 1. Grasp kinematics of the cooperative manipulation task.

The coordinate frame $O$ is chosen to coincide with the object's center of mass. Note that a wrench $\tilde{h}=\left(\tilde{f}^{T}, \tilde{t}^{T}\right)^{T}$ applied to an arbitrary location $\tilde{r} \in \mathbb{R}^{3}$ on the object (different from its center of mass) does not induce any rotational motion if $\tilde{t}=-S(\tilde{r}) \tilde{f}$, since the torque $\tilde{t}$ compensates the emerging torque due to the force $\tilde{f}$ and $\tilde{r} \neq 0$. However, the application of the same wrench $\tilde{h}$ about the object's center of mass induces actually a rotation of the object. Different from previous results [5], the choice of the coordinate system is in fact relevant to the wrench analysis and the object's center of mass is a physically consistent choice [8].

Problem statement: Find a static or dynamic load allocation strategy for the cooperative manipulation task. Namely, given a desired wrench $h_{o}^{d}$, assign suitable desired end-effector wrenches $h^{d}$ for the manipulator ensemble

- satisfying constraint (1), i.e. the applied end-effector wrenches implement the desired object wrench;

- taking into account the potentially heterogeneous payload capacities for the load distribution;

- accounting for dynamical (time-varying) constraints on the individual manipulator payload;

- minimizing internal wrenches that can possibly damage the manipulated object.

In the sequel we assume feasibility of the allocation problem, i.e. the desired wrench $h_{o}^{d}$ can always be implemented without exceeding the static and dynamic saturation limits of the manipulator ensemble.

\section{STATIC LOAD ALLOCATION}

For the static load allocation we aim at finding a parametrized generalized inverse $G^{+}$for inverting (1) such that

$$
h^{d}=G^{+}(\beta) h_{o}^{d},
$$

where parameters $\beta$ are defined below and where $h^{d}$ satisfies the equality constraint (1). To this end, we reformulate the load allocation problem as a constrained optimization problem and introduce weighting coefficients in order to tune the individual manipulator load. Let $\beta_{i} \in \mathbb{R}$ with $0<\beta_{i}<1$ denote the constant associated load distribution coefficient for the $i$-th manipulator. By imposing the normalization

$$
\sum_{i=1}^{N} \beta_{i}=1,0<\beta_{i}<1, i=1, \ldots, N
$$

the $\beta_{i}$ 's can be interpreted as the percentage of load distributed to the $i$-th manipulator.

\section{A. Constrained optimization problem}

The load distribution problem in (3) can be expressed as a constrained optimization problem according to

$$
\begin{array}{cl}
\min _{h^{d}} & h^{d^{T}} W h^{d} \\
\text { s.t. } & h_{o}^{d}=G h^{d}
\end{array}
$$

with the (constant) block-diagonal positive definite matrix $W=\operatorname{blockdiag}\left\{\frac{1}{\beta_{i}} I_{6}\right\}, i=1, \ldots, N$. The Lagrange Multiplier theorem [12] can be applied to derive an explicit, closed-form solution for the the desired end-effector wrenches $h_{i}^{d}$. Given $G$ in (2) and any $\beta_{i}$ satisfying (4), the unique optimal solution to (5) is given by:

$$
\left(\begin{array}{c}
f_{i}^{d} \\
t_{i}^{d}
\end{array}\right)=\beta_{i}\left[\begin{array}{cc}
I_{3}-\bar{S} K_{\Delta} S(\Delta) & \bar{S} K_{\Delta} \\
-K_{\Delta} S(\Delta) & K_{\Delta}
\end{array}\right]\left(\begin{array}{c}
f_{o}^{d} \\
t_{o}^{d}
\end{array}\right)
$$

with $\bar{S}=S(\Delta)-S\left(r_{i}\right)$, and $K_{\Delta}=\left(I_{3}+T\right)^{-1}$, $T=\left(I^{t}-S(\Delta)^{T} S(\Delta)\right)$ and

$$
\Delta=\sum_{i=1}^{N} \beta_{i} r_{i}
$$

The vector $\Delta \in \mathbb{R}^{3}$ points from the object's physical center of mass to its virtual center of mass (VCM) depicted with the coordinate system $O^{*}$ in Fig. 1 and matrix $T$ represents the weighted inertia tensor of the system of endeffectors with respect to its virtual center of mass, while $I^{t}=\sum_{i=1}^{N} \beta_{i} S\left(r_{i}\right) S^{T}\left(r_{i}\right)$ is the weighted inertia tensor with respect to the center of mass of the object.

\section{B. Choice of the weighting coefficients}

The choice of the weighting coefficients $\beta_{i}$ is initially arbitrary. However, as indicated in (7), they determine the displacement $\Delta$ between the VCM and the physical center of mass (see Fig. 1). By combining (4) and (7) it is straightforward to verify that the VCM is contained in the convex hull of the grasp points $r_{i}$. The VCM is closer to 
the end-effectors with the largest weight $\beta_{i}$, i.e. the ones with comparatively larger virtual masses. For $\beta_{1}>\beta_{2}, \beta_{3}$ the resulting location of the VCM is illustrated in Fig 1.

To understand the consequences of selecting $\Delta \neq 0$, notice that in all cases the ensemble of allocated (desired) endeffector wrenches $h^{d}$ produces the desired wrench applied to the object $h_{o}^{d}$ as required in (1). However, when $\Delta \neq 0$, the resulting object wrench $h_{o}^{d}$ is not applied to its physical center of mass but to its virtual center of mass. This means that an additional (undesired) torque is applied to the object's physical center of mass, which is given by

$$
t_{\Delta}=S(\Delta) f_{o}^{d}
$$

Clearly, the torque $t_{\Delta}$ vanishes for $\Delta=0$. In general this additional torque is considered to be a disturbance and thus limits the admissible choices of the weighting coefficients $\beta_{i}$. The emerging question is whether one can find $\beta_{i}$ such that $\Delta=0$. An answer is given in the next result.

Proposition 1: The set of load distribution weights $\beta_{i}$ leading to $\Delta=0$ satisfies the following:

- it has exactly one solution for $N=2$ with the grasp point vector $r_{1}=-c r_{2}$ for any $c \in \mathbb{R}^{+}$, otherwise it is empty (i.e. for almost all grasp point vectors $r_{1}, r_{2}$ ).

- has exactly one solution for $N=3$ and the grasp point vectors $r_{i}$ spanning $\mathbb{R}^{3}$.

- has multiple solutions for $N>3$ and the grasp point vectors $r_{i}$ spanning $\mathbb{R}^{3}$.

Proof: The result follows from reformulating (7) as a system of linear equations in $\beta_{i}$ and requiring $\Delta=0$, namely:

$$
\Delta=\underbrace{\left[\begin{array}{lll}
r_{1} & \ldots & r_{N}
\end{array}\right]}_{R:=}\left[\begin{array}{c}
\beta_{1} \\
\vdots \\
\beta_{N}
\end{array}\right]=\left[\begin{array}{l}
0 \\
0 \\
0
\end{array}\right] .
$$

The vector $\beta=\left[\beta_{1}, \ldots, \beta_{N}\right]^{T}$ needs to lie in the null space of matrix $R$ in (9). For $N=2$, since $R \in \mathbb{R}^{3 \times 2}$, this null space is empty for almost all selections of vectors $r_{i}$. If $N=2$ and $r_{1}=-c r_{2}$ for some $c>0$ then one may select $\left[\beta_{1}, \beta_{2}\right]^{T}=\frac{1}{1+c}[1, c]^{T}$. For $N=3$ the result follows similarly because $r_{i}$ spanning $\mathbb{R}^{3}$ implies that $R$ is non-singular. Finally, due to the manipulator redundancy for $N>3$, the solution to (9) is no longer unique.

In case of $\Delta=0$, the expression for the generalized inverse in (3) as presented in (6) simplifies to

$$
G_{0}^{+}(\beta)=\left[\begin{array}{cc}
\beta_{1} I_{3} & -\beta_{1} S\left(r_{1}\right) K_{\Delta} \\
0_{3} & \beta_{1} K_{\Delta} \\
\vdots & \vdots \\
\beta_{N} I_{3} & -\beta_{N} S\left(r_{N}\right) K_{\Delta} \\
0_{3} & \beta_{N} K_{\Delta}
\end{array}\right] \in \mathbb{R}^{6 N \times 6}
$$

with $K_{\Delta}=\left(I_{3}+I^{t}\right)^{-1}$. Matrix $G_{0}^{+}(\beta)$ in (10) is a right inverse of the grasp matrix, i.e. $G G_{0}^{+}(\beta)=I$. Moreover, $G_{0}^{+}(\beta)$ represents a generalization of the Moore-Penrose pseudo-inverse, as presented in [7], to the case of heterogeneous payload capacities.

\section{Illustration of the load allocation strategy}

Consider the cooperative manipulation task example for $N=2$ and $r_{1}=-r_{2}=(-1,0,0)^{T}$ m depicted in Fig. 2.

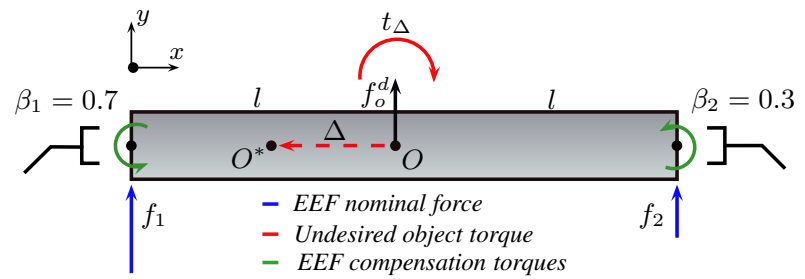

Fig. 2. Manipulation task example for $N=2$.

Let $f_{o}^{d}=(0,10,0)^{T} N$ and $t_{o}^{d}=0$ and choose the load allocation strategy according to $\beta_{1}=0.7$ and $\beta_{2}=0.3$. Employing (6) for computing the desired end-effector wrenches yields $f_{1}^{d}=(0,6.1,0)^{T} \mathrm{~N}$ and $f_{2}^{d}=(0,3.9,0)^{T} \mathrm{~N}$. Since $\Delta=0.4 \mathrm{~m}$, the allocated end-effector forces themselves would induce a resulting torque about the object's physical center of mass. This explains why the allocation strategy (6) yields the allocated torques $t_{1}^{d}=(0,0,1.52)^{T} \mathrm{Nm}$ and $t_{2}^{d}=(0,0,0.65)^{T} \mathrm{Nm}$ for the allocated end-effector torques, that are needed to compensate the disturbing torque $t_{\Delta}$.

Note further that the resulting undesired torque $t_{\Delta}=(0,0,-2.2)^{T} \mathrm{Nm}$ can either be compensated by the values of $t_{1}^{d}$ and $t_{2}^{d}$ indicated above or by any other combination of $t_{1}^{d}$ and $t_{2}^{d}$ summing up to $t_{\Delta}$. For example $t_{1}^{d}=(0,0,1.1)^{T} \mathrm{Nm}$ and $t_{2}^{d}=(0,0,1.1)^{T} \mathrm{Nm}$ or $t_{1}^{d}=(0,0,2.2)^{T} \mathrm{Nm}$ and $t_{2}^{d}=(0,0,0)^{T} \mathrm{Nm}$ represent valid load distributions, too. This observation indicates that there are additional degrees of freedom available for the load distribution which are not incorporated in the weighting coefficients $\beta$. It turns out that our choice of an equivalent weighting of force and torque components restricts artificially the available degrees of freedom for the load allocation.

\section{Allocation analysis for $\Delta \neq 0$}

In this section we investigate in more detail the impact of $\Delta \neq 0$ and the implicitly performed compensation through the allocation strategy (6). By introducing

$$
h_{\Delta}=\left[\begin{array}{l}
f_{\Delta} \\
t_{\Delta}
\end{array}\right]=\left[\begin{array}{c}
S(\Delta) K_{\Delta} t_{o}^{d} \\
S(\Delta) f_{o}^{d}
\end{array}\right]
$$

we rearrange the terms in (6) in order to obtain

$$
h^{d}=G_{0}^{+}(\beta) h_{o}^{d}+C(\lambda) h_{\Delta} .
$$

The additional weighting coefficients $\lambda=\left(\lambda_{1}, \ldots, \lambda_{N}\right)^{T}$ with $\lambda_{i} \in \mathbb{R}$ satisfying $0 \leq \lambda_{i} \leq 1, i=1, \ldots, N$ parametrize the compensation matrix

$$
C(\lambda)=\left[\begin{array}{cc}
\beta_{1} I_{3} & -\lambda_{1} \bar{S}_{\lambda} K_{\lambda} \\
0_{3} & \lambda_{1} K_{\lambda} \\
\vdots & \vdots \\
\beta_{N} I_{3} & -\lambda_{N} \bar{S}_{\lambda} K_{\lambda} \\
0_{3} & \lambda_{N} K_{\lambda}
\end{array}\right] \in \mathbb{R}^{6 N \times 6}
$$


TABLE I

SIMULATION RESULTS

\begin{tabular}{|c|c|c|c|c|c|c|c|}
\hline \multirow{2}{*}{\multicolumn{2}{|c|}{ Wrench }} & \multicolumn{3}{|c|}{ Force } & \multicolumn{3}{|c|}{ Torque } \\
\hline & & $f_{x}$ & $f_{y}$ & $f_{z}$ & $t_{x}$ & $t_{y}$ & $t_{z}$ \\
\hline & $h_{o}^{d}$ & 0 & 10 & 0 & 0 & 0 & 0 \\
\hline \multirow{2}{*}{ A } & $h_{1}^{d}$ & 0 & 6.1 & 0 & 0 & 0 & 1.52 \\
\hline & $h_{2}^{d}$ & 0 & 3.9 & 0 & 0 & 0 & 0.65 \\
\hline \multirow{2}{*}{ B } & $h_{1}^{d}$ & 0 & 6 & 0 & 0 & 0 & 1 \\
\hline & $h_{2}^{d}$ & 0 & 4 & 0 & 0 & 0 & 1 \\
\hline \multirow{2}{*}{$\mathrm{C}$} & $h_{1}^{d}$ & 0 & 7 & 0 & 0 & 0 & 4 \\
\hline & $h_{2}^{d}$ & 0 & 3 & 0 & 0 & 0 & 0 \\
\hline
\end{tabular}

The matrices $\bar{S}_{\lambda}$ and $K_{\lambda}$ are obtained by replacing $\beta$ with $\lambda$ in $\bar{S}$ and $K_{\Delta}$ defined below equation (6). It is easily verified that $C(\lambda) h_{\Delta}$ in (12) vanishes for $\Delta=0$. In this sense, we interpret $C(\lambda) h_{\Delta}$ as a compensating wrench ensuring the equality constraint (1) whenever $\Delta \neq 0$ and $G_{0}^{+}(\beta) h_{o}^{d}$ as the nominally allocated end-effector wrench. As motivated through the observations in the previous section, the compensation matrix $C$ parameterized by $\lambda$ provides additional degrees of freedom for the load distribution, which are discussed in the following example.

Example: Consider again the manipulation task depicted in Fig. 2 with $\beta_{1}=0.7$ and $\beta_{2}=0.3$. Table $\mathrm{I}^{1}$ shows the end-effector wrench obtained from (12) with the comparison between three different compensation strategies:

A. $\lambda_{i}=\beta_{i}, i=1,2$. The resulting wrench is equivalent to the one obtained in Section III-C;

B. $\lambda_{1}=\lambda_{2}=0.5$. We choose a uniform distribution of the compensation wrench: it follows that $t_{1_{z}}=t_{2_{z}}$.

C. $\lambda_{1}=1, \lambda_{2}=0$. Only manipulator 1 is assigned the compensation task, i.e. $t_{1_{z}} \neq 0$ and $t_{2 z}=0$.

According to (12), the resulting force in cases A and B is affected by the distribution of $h_{\Delta}$ to the individual manipulator wrenches. This is due to the fact that we set equal weights for the force and torque components. In addition, notice that the new formulation of the end-effector wrench does not always correspond to the minimization criterion imposed by (5). In general, if $\lambda_{i} \neq \beta_{i}$ the resulting wrench (12) will be different from the solution of the optimization problem (5).

Remark 1: Notice that any load distribution in this example satisfies (1). The torques do not contribute to the effective motion of the object and are therefore internal.

\section{DYNAMIC LOAD ALLOCATION}

In this section we extend the presented load distribution scheme in view of existing time-varying payload limits of the individual manipulators. In the previous sections, the payload limits were implicitly incorporated into the allocator by choosing appropriate weighting coefficients $\beta$. Existing load allocation schemes [9] allow to impose explicit limits on the allocated manipulator payload. We firstly present a model

\footnotetext{
${ }^{1}$ Green is related to compensation terms, blue stands for allocation and magenta indicates terms that include both allocation and compensation.
}

for the admissible manipulator payload before proposing a dynamic load allocation strategy accounting for the timevarying manipulator capacities.

\section{A. Payload capacity model}

For the analysis of the admissible individual manipulator payload, we assume that each manipulator exhibits 6 degrees of freedom characterized by the joint angles $q_{i} \in \mathbb{R}^{6}$ and that the joint torque limits are given by the constant vector $\bar{\tau}_{i} \in \mathbb{R}^{6}$. In the quasi-static case the admissible end-effector payload of the individual manipulator is a function of the configuration according to

$$
\bar{h}_{i}=J_{i}^{-T}\left(q_{i}\right) \bar{\tau}_{i}
$$

wherein $J_{i}$ is the individual manipulator Jacobian. Moreover, differentiating (14) leads to additional constraints on the rate of change of the admissible payload, i.e.

$$
\frac{d}{d t}\left\{\bar{h}_{i}\right\}=J_{i}^{-T}\left(q_{i}\right) J_{i}^{-1}\left(q_{i}\right) G_{i}^{T} \dot{x}_{o}^{d}
$$

with $G_{i}^{T}$ being the $i$-th block of the transpose grasp matrix and $\dot{x}_{o}^{d}$ the desired velocity of the object.

Remark 2: We can model (14) and (15) as state dependent magnitude and rate saturation limits on the end-effector wrench. However, since dealing with state-dependent constraints is quite involved, in the following we only consider them as time-varying saturation limits, leaving the extension to explicit dependency on the state variables to future work.

\section{B. Dynamic allocator}

In light of Remark 2, we introduce a suitable dynamic allocator in the load distribution block in order to deal with the additional saturation limits. The allocator modifies the static wrench (12) exploiting the strongly redundant nature of the cooperative system: indeed, it acts on the wrenches belonging to the kernel of the grasp matrix, i.e. the wrenches that are not related to the effective motion of the object. We only focus here on the case of magnitude saturation, refer to [9] for more general dynamic allocators involving rate constraints. Consider the dynamically allocated end-effector wrench $\tilde{h}^{d}$ defined by

$$
\tilde{h}^{d}=h^{d}+G_{\perp} w
$$

wherein $G_{\perp}=\left(I-G^{+} G\right)$ and $w$ is the output of a proper dynamic allocator. It is straightforward to verify that $G G_{\perp}=0$ because $G G^{+}=I$, thus the additional signal $w$ acts on the wrenches belonging to the kernel of the grasp matrix. Therefore, the dynamic allocator remains invisible to the subsequent blocks of the control scheme and the final distributed wrench $\tilde{h}^{d}$ is such that $G \tilde{h}^{d}=G h^{d}$, i.e. the geometric constraint (1) is still satisfied.

Cost function: Assuming that $h^{d}$ in (16) is a fixed parameter, define the cost function

$$
V(w)=\left(\tilde{h}^{d}\right)^{T} \bar{U}\left(\tilde{h}^{d}, \bar{h}\right) \tilde{h}^{d},
$$

wherein $\tilde{h}^{d}$ is the stacked vector of the desired endeffector wrench given by (16) and $\bar{U}\left(\tilde{h}^{d}, \bar{h}\right)$ represents a 
non-constant matrix function taking into account the (timevarying) magnitude saturation limits (14). Given a small constant $\epsilon \in(0,1)$, a good selection for the weighting matrix is given in [9] and corresponds to:

$$
\bar{U}\left(\tilde{h}^{d}, \bar{h}\right)=\left(\operatorname{diag}\left((1+\epsilon) \bar{h}-\operatorname{abs}\left(\operatorname{sat}\left(\tilde{h}^{d}\right)\right)\right)\right)^{-1},
$$

with the vectors $\bar{h}, \quad \operatorname{sat}\left(\tilde{h}^{d}\right) \in \mathbb{R}^{6 N}$ given by ${ }^{2} \quad \bar{h}=\left[\bar{h}_{1}^{T}, \ldots, \bar{h}_{N}^{T}\right]^{T} \quad$ and $\operatorname{sat}\left(\tilde{h}^{d}\right)=\left[\operatorname{sat}\left(\tilde{h}_{1}^{d}\right)^{T}, \ldots, \operatorname{sat}\left(\tilde{h}_{N}^{d}\right)^{T}\right]^{T}$. The saturation function for each end-effector is defined as

$$
\operatorname{sat}\left(\tilde{h}_{i}^{d}\right):=\operatorname{diag}\left\{\sigma\left(\tilde{h}_{i_{1}}^{d} / \bar{h}_{i_{1}}\right), \ldots, \sigma\left(\tilde{h}_{i_{6}}^{d} / \bar{h}_{i_{6}}\right)\right\} \bar{h}_{i}
$$

and the unit saturation function is defined by $\sigma(s):=\operatorname{sign}(s) \min \{|s|, 1\} \quad, \forall s \in \mathbb{R}$. The weighting matrix (18) is suitable for our goal since its diagonal terms increase as the argument approaches the respective saturation level. Therefore, it allows us adjusting online the selection of the end-effectors to be promoted or penalized in order to keep them far from their saturation limits. Moreover, notice that $\bar{U}\left(\tilde{h}^{d}, \bar{h}\right) \geq\left(\operatorname{diag}((1+\epsilon) \bar{h})^{-1}\right.$, thus (18) is a uniformly positive definite matrix.

Allocator dynamics: According to [9], we design the dynamics of the allocator based on the cost function (17) and a symmetric positive definite $K$. The resulting non-linear dynamic allocator is

$$
\begin{aligned}
& \dot{w}=-K G_{\perp}^{T} \bar{U}\left(\tilde{h}^{d}, \bar{h}\right) \tilde{h}^{d} \\
& \tilde{h}^{d}=h^{d}+G_{\perp} w
\end{aligned}
$$

In our case the magnitude saturation limits are not constant. However, due to the limited rate of change of the maximal payload represented by (15), we can consider $\bar{h}_{i}(t)$ in (20) as slowly time-varying parameters. The extended load distribution block with both static and dynamic load allocation is depicted in Fig. 3.

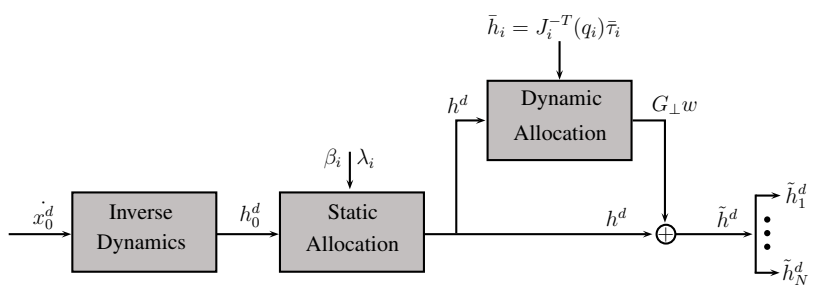

Fig. 3. Illustration of the load allocation block scheme.

\section{EXPERIMENTAL EVALUATION}

\section{A. Experimental setup}

The experimental setup involving two anthropomorphic manipulators with 7 degrees of freedom each, with both endeffectors rigidly grasping a metal beam with a length of $1 \mathrm{~m}$ and a quadratic profile of $1.5 \mathrm{~mm}$ is depicted in Fig. 4 .

The object coordinate frame coincides with the center of mass of the beam and the end-effector grasping points are

\footnotetext{
${ }^{2}$ For notational convenience we do not explicitly indicate the time dependency of the saturation limits.
}

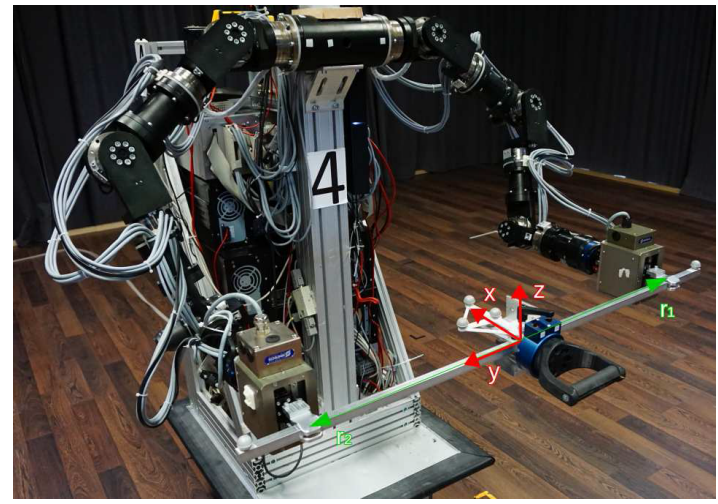

Fig. 4. Experimental setup with two robotic manipulators.

$r_{1}=(0,-0.4,0)^{T} \mathrm{~m}, r_{2}=(0,+0.4,0)^{T} \mathrm{~m}$. The overall mass of the object is $m_{o}=3.405 \mathrm{~kg}$; with the gravity constant $g=9.81 \frac{\mathrm{m}}{\mathrm{s}^{2}}$. The desired wrench for holding the object at rest is $h_{o}=\left(0,0, m_{o} g, 0,0,0\right)^{T}$. Each arm is equipped with a JR3 67M25 6-dimentional force/torque sensor to measure the actual end-effector wrench.

\section{B. Comparison between different load distributions}

In the experimental study we compare three load distribution strategies obtained from different combinations of the nominal and compensation weights in (12) for holding the object at rest. Thus, only the z-component of the resulting force and the torque about the $x$-axis is relevant. The endeffector wrenches for a uniform (equal) load distribution, i.e. $\beta_{i}=\lambda_{i}=0.5, i=1,2$ are plotted in Fig. 5 .
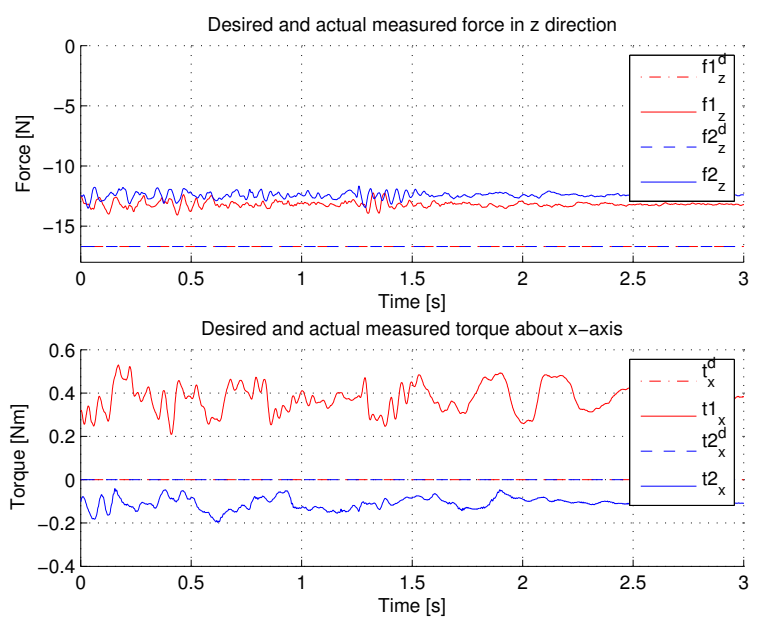

Fig. 5. Desired and measured end-effector force/torque for $\left(\beta_{1}, \beta_{2}\right)=(0.5,0.5),\left(\lambda_{1}, \lambda_{2}\right)=(0.5,0.5)$.

The manipulators exert approximately the same force in the $z$-direction. Since the choice of the weighting factors implies $\Delta=0$, the torque component is close to zero. The gap between real and desired values of the end-effector torque is explained by a limited calibration accuracy of the arm kinematics and a resulting (static) error in the compensation of the end-effector mass. The variation of the 
force/torque signals is due to the interaction of the rigidly coupled, impedance-controlled end-effectors. In summary, the load distribution in Fig. 5 is considered appropriate for homogeneous manipulators with equal payload capacities.

The manipulator wrenches for a heterogeneous load distribution strategy with $\beta_{1}=\lambda_{1}=0.7$ and $\beta_{2}=\lambda_{2}=0.3$ are depicted in Fig. 6.
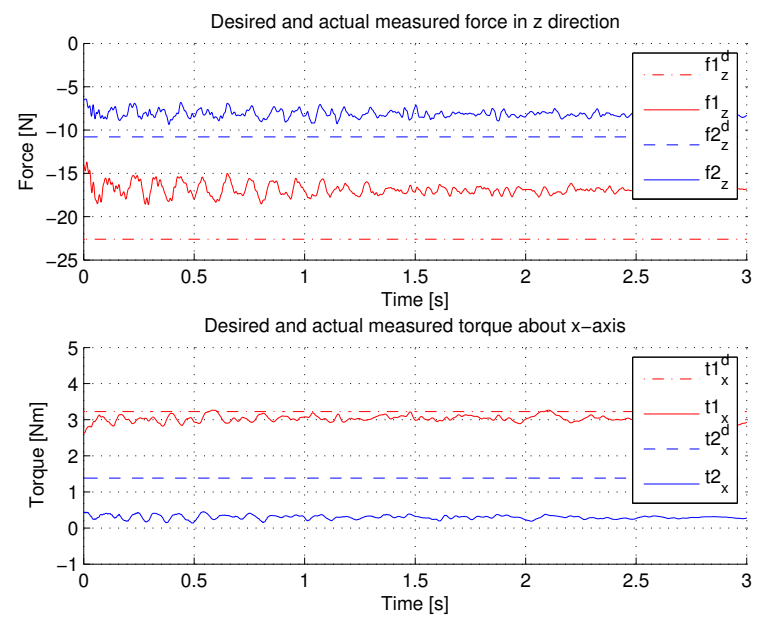

Fig. 6. Desired and measured end-effector force/torque for $\beta_{1}=\lambda_{1}=0.7$ and $\beta_{2}=\lambda_{2}=0.3$.

The measured force and torque signals agree with the desired wrenches while the majority of the load is carried by manipulator 1 . The choice of the weighting factors implies $\Delta \neq 0$ which according to (8) gives rise to a torque acting on the object. This is compensated by the additional (non-zero) torques at the end-effectors. Given the specific selection of the weights $\lambda_{i}$, manipulator 2 applies a lower compensation torque than manipulator 1 .
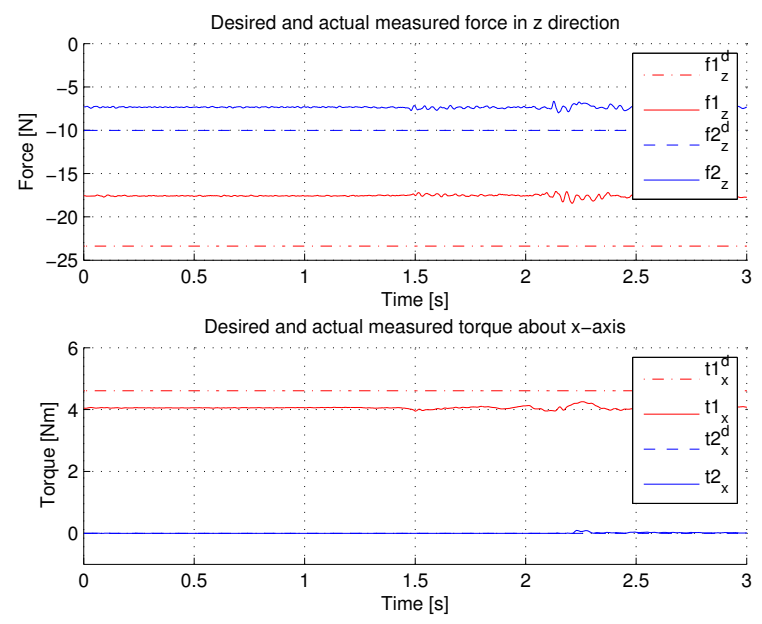

Fig. 7. End-effector desired and measured force/torque for $\left(\beta_{1}, \beta_{2}\right)=(0.7,0.3),\left(\lambda_{1}, \lambda_{2}\right)=(1,0)$.

The effect of heterogeneous compensation weights for $\lambda_{1}=1, \lambda_{2}=0$ and again $\beta_{1}=0.7$ and $\beta_{2}=0.3$ is illustrated in Fig. 7. Similar to case $\mathrm{C}$ in Table I, the undesired torque due to $\Delta \neq 0$ is now entirely compensated by manipulator 1 , as expected.

Discussion: The presented experimental study clearly highlights the relevance of our findings for static load allocation. The impact of different load strategies is illustrated best while keeping the object at rest, although the extension to arbitrary motions is straightforward. Note that the approach is also suitable for dynamic load allocation, its experimental evaluation is not presented here because of space constraints.

\section{CONCLUSION AND OUTLOOK}

In this paper we present a novel approach to the load distribution in cooperative multi-robot manipulation tasks. We present a load allocation strategy addressing heterogeneous payload capacities of the manipulators in case of constant or slowly time-varying load constraints. The relevance of our findings is evaluated in an experiment study involving two anthropomorphic manipulators. Future work includes a thorough analysis of the physically consistent degrees of freedom for the load distribution in terms of their connection with internal forces and an extended stability study.

\section{ACKNOWLEDGMENT}

The research leading to these results has received funding from the European Union Seventh Framework Programme FP7/2007-2013 under grant agreement n 601165 of the project "WEARHAP WEARable HAPtics for humans and robots".

\section{REFERENCES}

[1] T. A. Johansen and T. I. Fossen, "Control allocationa survey," Automatica, vol. 49, no. 5, pp. $1087-1103,2013$.

[2] N. Hogan, "Impedance control: An approach to manipulation," in American Control Conference, 1984, pp. 304-313, June 1984.

[3] T. Alberts and D. Soloway, "Force control of a multi-arm robot system," in Robotics and Automation, 1988. Proceedings., 1988 IEEE International Conference on, pp. 1490-1496 vol.3, Apr 1988.

[4] I. D. Walker, R. A. Freeman, and S. I. Marcus, "Analysis of motion and internal loading of objects grasped by multiple cooperating manipulators," The International Journal of Robotics Research, vol. 10, no. 4, pp. 396-409, 1991.

[5] R. Bonitz and T. Hsia, "Force decomposition in cooperating manipulators using the theory of metric spaces and generalized inverses," in Robotics and Automation, 1994. Proceedings., 1994 IEEE International Conference on, pp. 1521-1527 vol.2, May 1994.

[6] D. Williams and O. Khatib, "The virtual linkage: a model for internal forces in multi-grasp manipulation," in Robotics and Automation, 1993. Proceedings., 1993 IEEE International Conference on, pp. 1025-1030 vol.1, 1993.

[7] J. Chung, B.-J. Yi, and W. Kim, "Analysis of internal loading at multiple robotic systems," Journal of Mechanical Science and Technology, vol. 19, no. 8, pp. 1554-1567, 2005.

[8] S. Erhart and S. Hirche, "Internal force analysis and load distribution for cooperative multi-robot manipulation," IEEE Transactions on Robotics, to appear, http://www.itr.ei.tum.de, 2015.

[9] L. Zaccarian, "Dynamic allocation for input redundant control systems," Automatica, vol. 45, no. 6, pp. 1431 - 1438, 2009.

[10] S. Schneider and J. Cannon, R.H., "Object impedance control for cooperative manipulation: theory and experimental results," Robotics and Automation, IEEE Transactions on, vol. 8, pp. 383-394, Jun 1992

[11] B. Siciliano and O. Khatib, eds., Springer Handbook of Robotics. Berlin, Heidelberg: Springer, 2008.

[12] J. Nocedal and S. Wright, Numerical Optimization. Springer Series in Operations Research and Financial Engineering, Springer New York, 2006. 